\title{
ON HYPERCOMPLEX PSEUDO-HERMITIAN MANIFOLDS
}

\author{
KOSTADIN GRIBACHEV \\ Faculty of Mathematics and Informatics, University of Plovdiv, \\ 236 Bulgaria Blvd., Plovdiv 4003, Bulgaria \\ E-mail: costas@uni-plovdiv.bg \\ MANCHO MANEV \\ Faculty of Mathematics and Informatics, University of Plovdiv, \\ 236 Bulgaria Blvd., Plovdiv 4003, Bulgaria \\ E-mail: mmanev@uni-plovdiv.bg \\ STANCHO DIMIEV \\ Institute of Mathematics and Informatics, Bulgarian Academy of Sciences, \\ Acad. G. Bonchev Str., Bl. 8, Sofia 1113, Bulgaria, \\ E-mail:sdimiev@math.bas.bg

\begin{abstract}
The class of the hypercomplex pseudo-Hermitian manifolds is considered. The flatness of the considered manifolds with the 3 parallel complex structures is proved. Conformal transformations of the metrics are introduced. The conformal invariance and the conformal equivalence of the basic types manifolds are studied. A
\end{abstract} \\ known example is characterized in relation to the obtained results.
}

\section{Introduction}

This paper is a continuation of the same authors's paper 4 which is inspired by the seminal work 1 of D. V. Alekseevsky and S. Marchiafava. We follow a parallel direction including skew-Hermitian metrics with respect to the almost hypercomplex structure.

In the first section we give some necessary facts concerning the almost hypercomplex pseudo-Hermitian manifolds introduced in 4 .

In the second one we consider the special class of (integrable) hypercomplex pseudo-Hermitian manifolds, namely pseudo-hyper-Kähler manifolds. Here we expose the proof of the mentioned in 4 statement that each pseudohyper-Kähler manifold is flat.

The third section is fundamental for this work. A study of the group of conformal transformations of the metric is initiated here. The conformal invariant classes and the conformal equivalent class to the class of the pseudohyper-Kähler manifolds are found.

Finally, we characterize a known example in terms of the conformal transformations.

manev12: submitted to World Scientific on August 17, 2021 


\section{Preliminaries}

\subsection{Hypercomplex pseudo-Hermitian structures in a real vector space}

Let $V$ be a real $4 n$-dimensional vector space. By $\left\{\frac{\partial}{\partial x^{i}}, \frac{\partial}{\partial y^{i}}, \frac{\partial}{\partial u^{i}}, \frac{\partial}{\partial v^{i}}\right\}, i=$ $1,2, \ldots, n$, is denoted a (local) basis on $V$. Each vector $x$ of $V$ is represented in the mentioned basis as follows

$$
x=x^{i} \frac{\partial}{\partial x^{i}}+y^{i} \frac{\partial}{\partial y^{i}}+u^{i} \frac{\partial}{\partial u^{i}}+v^{i} \frac{\partial}{\partial v^{i}} .
$$

A standard complex structure on $V$ is defined as in 5 :

$$
\begin{aligned}
& J_{1} \frac{\partial}{\partial x^{i}}=\frac{\partial}{\partial y^{i}}, \quad J_{1} \frac{\partial}{\partial y^{i}}=-\frac{\partial}{\partial x^{i}}, J_{1} \frac{\partial}{\partial u^{i}}=-\frac{\partial}{\partial v^{i}}, J_{1} \frac{\partial}{\partial v^{i}}=\frac{\partial}{\partial u^{i}} ; \\
& J_{2} \frac{\partial}{\partial x^{i}}=\frac{\partial}{\partial u^{i}}, \quad J_{2} \frac{\partial}{\partial y^{i}}=\frac{\partial}{\partial v^{i}}, \quad J_{2} \frac{\partial}{\partial u^{i}}=-\frac{\partial}{\partial x^{i}}, J_{2} \frac{\partial}{\partial v^{i}}=-\frac{\partial}{\partial y^{i}} ; \\
& J_{3} \frac{\partial}{\partial x^{i}}=-\frac{\partial}{\partial v^{i}}, J_{3} \frac{\partial}{\partial y^{i}}=\frac{\partial}{\partial u^{i}}, \quad J_{3} \frac{\partial}{\partial u^{i}}=-\frac{\partial}{\partial y^{i}}, J_{3} \frac{\partial}{\partial v^{i}}=\frac{\partial}{\partial x^{i}} .
\end{aligned}
$$

The following properties about $J_{\alpha}$ are direct consequences of (2)

$$
\begin{aligned}
& J_{1}^{2}=J_{2}^{2}=J_{3}^{2}=-I d, \\
& J_{1} J_{2}=-J_{2} J_{1}=J_{3}, \quad J_{2} J_{3}=-J_{3} J_{2}=J_{1}, \quad J_{3} J_{1}=-J_{1} J_{3}=J_{2} .
\end{aligned}
$$

If $x \in V$, i.e. $x\left(x^{i}, y^{i}, u^{i}, v^{i}\right)$ then according to (2) and (3) we have

$$
J_{1} x\left(-y^{i}, x^{i}, v^{i},-u^{i}\right), \quad J_{2} x\left(-u^{i},-v^{i}, x^{i}, y^{i}\right), \quad J_{3} x\left(v^{i},-u^{i}, y^{i},-x^{i}\right) .
$$

Definition $1.1(\underline{1})$ A triple $H=\left(J_{1}, J_{2}, J_{3}\right)$ of anticommuting complex structures on $V$ with $J_{3}=J_{1} J_{2}$ is called a hypercomplex structure on $V$;

A bilinear form $f$ on $V$ is defined as ordinary, $f: V \times V \rightarrow \mathbb{R}$. We denote by $\mathcal{B}(V)$ the set of all bilinear forms on $V$. Each $f$ is a tensor of type $(0,2)$, and $\mathcal{B}(V)$ is a vector space of dimension $16 n^{2}$.

Let $J$ be a given complex structure on $V$. A bilinear form $f$ on $V$ is called Hermitian (respectively, skew-Hermitian) with respect to $J$ if the identity $f(J x, J y)=f(x, y)$ (respectively, $f(J x, J y)=-f(x, y)$ holds true.

Definition $1.2(\mathbf{1})$ A bilinear form $f$ on $V$ is called an Hermitian bilinear form with respect to $H=\left(J_{\alpha}\right)$ if it is Hermitian with respect to any complex structure $J_{\alpha}, \alpha=1,2,3$, i.e.

$$
f\left(J_{\alpha} x, J_{\alpha} y\right)=f(x, y) \quad \forall x, y \in V .
$$

We denote by $L_{0}=\mathcal{B}_{H}(V)$ the set of all Hermitian bilinear forms on $V$. The notion of pseudo-Hermitian bilinear forms is introduced by the following 
Definition 1.3 (4) A bilinear form $f$ on $V$ is called a pseudo-Hermitian bilinear form with respect to $H=\left(J_{1}, J_{2}, J_{3}\right)$, if it is Hermitian with respect to $J_{\alpha}$ and skew-Hermitian with respect to $J_{\beta}$ and $J_{\gamma}$, i.e.

$$
f\left(J_{\alpha} x, J_{\alpha} y\right)=-f\left(J_{\beta} x, J_{\beta} y\right)=-f\left(J_{\gamma} x, J_{\gamma} y\right)=f(x, y) \quad \forall x, y \in V,
$$

where $(\alpha, \beta, \gamma)$ is a circular permutation of $(1,2,3)$.

We denote $f \in L_{\alpha} \subset \mathcal{B}(V)(\alpha=0,1,2,3)$ when $f$ satisfies the conditions (5) and (6), respectively.

In 1 is introduced a pseudo-Euclidian metric $g$ with signature $(2 n, 2 n)$ as follows

$$
g(x, y):=\sum_{i=1}^{n}\left(-x^{i} a^{i}-y^{i} b^{i}+u^{i} c^{i}+v^{i} d^{i}\right),
$$

where $x\left(x^{i}, y^{i}, u^{i}, v^{i}\right), y\left(a^{i}, b^{i}, c^{i}, d^{i}\right) \in V, i=1,2, \ldots, n$. This metric satisfies the following properties

$$
g\left(J_{1} x, J_{1} y\right)=-g\left(J_{2} x, J_{2} y\right)=-g\left(J_{3} x, J_{3} y\right)=g(x, y) .
$$

This means that the pseudo-Euclidean metric $g$ belongs to $L_{1}$.

The form $g_{1}: g_{1}(x, y)=g\left(J_{1} x, y\right)$ coincides with the Kähler form $\Phi$ which is Hermitian with respect to $J_{\alpha}$, i.e.

$$
\Phi\left(J_{\alpha} x, J_{\alpha} y\right)=\Phi(x, y), \quad \alpha=1,2,3, \quad \Phi \in L_{0} .
$$

The attached to $g$ associated bilinear forms $g_{2}: g_{2}(x, y)=g\left(J_{2} x, y\right)$ and $g_{3}: g_{3}(x, y)=g\left(J_{3} x, y\right)$ are symmetric forms with the properties

$$
\begin{aligned}
& -g_{2}\left(J_{1} x, J_{1} y\right)=-g_{2}\left(J_{2} x, J_{2} y\right)=g_{2}\left(J_{3} x, J_{3} y\right)=g_{2}(x, y), \\
& -g_{3}\left(J_{1} x, J_{1} y\right)=g_{3}\left(J_{2} x, J_{2} y\right)=-g_{3}\left(J_{3} x, J_{3} y\right)=g_{3}(x, y),
\end{aligned}
$$

i.e. $g_{2} \in L_{3}, g_{3} \in L_{2}$.

It follows that the Kähler form $\Phi$ is Hermitian regarding $H$ and the metrics $g, g_{2}, g_{3}$ are pseudo-Hermitian of different types with signature $(2 n, 2 n)$.

Now we recall the following notion:

Definition $1.4\left({ }^{4}\right)$ The structure $(H, G):=\left(J_{1}, J_{2}, J_{3}, g, \Phi, g_{2}, g_{3}\right)$ is called a hypercomplex pseudo-Hermitian structure on $V$.

\subsection{Structural tensors on an almost $(H, G)$-manifold}

Let $(M, H)$ be an almost hypercomplex manifold 1 . We suppose that $g$ is a symmetric tensor field of type $(0,2)$. If it induces a pseudo-Hermitian inner product in $T_{p} M, p \in M$, then $g$ is called a pseudo-Hermitian metric on $M$.

manev12: submitted to World Scientific on August 17, 2021 
The structure $(H, G):=\left(J_{1}, J_{2}, J_{3}, g, \Phi, g_{2}, g_{3}\right)$ is called an almost hypercomplex pseudo-Hermitian structure on $M$ or in short an almost $(H, G)$-structure on $M$. The manifold $M$ equipped with $H$ and $G$, i.e. $(M, H, G)$, is called an almost hypercomplex pseudo-Hermitian manifold, or in short an almost $(H, G)$-manifold. 4

The 3 tensors of type $(0,3) F_{\alpha}: F_{\alpha}(x, y, z)=g\left(\left(\nabla_{x} J_{\alpha}\right) y, z\right), \alpha=1,2,3$, where $\nabla$ is the Levi-Civita connection generated by $g$, is called structural tensors of the almost $(H, G)$-manifold. 4

The structural tensors satisfy the following properties:

$$
\begin{gathered}
F_{1}(x, y, z)=F_{2}\left(x, J_{3} y, z\right)+F_{3}\left(x, y, J_{2} z\right), \\
F_{2}(x, y, z)=F_{3}\left(x, J_{1} y, z\right)+F_{1}\left(x, y, J_{3} z\right), \\
F_{3}(x, y, z)=F_{1}\left(x, J_{2} y, z\right)-F_{2}\left(x, y, J_{1} z\right) ; \\
F_{1}(x, y, z)=-F_{1}(x, z, y)=-F_{1}\left(x, J_{1} y, J_{1} z\right), \\
F_{2}(x, y, z)=F_{2}(x, z, y)=F_{2}\left(x, J_{2} y, J_{2} z\right), \\
F_{3}(x, y, z)=F_{3}(x, z, y)=F_{3}\left(x, J_{3} y, J_{3} z\right) .
\end{gathered}
$$

Let us recall the Nijenhuis tensors $N_{\alpha}(X, Y)=\frac{1}{2}\left[\left[J_{\alpha}, J_{\alpha}\right]\right](X, Y)$ for almost complex structures $J_{\alpha}$ and $X, Y \in \mathfrak{X}(M)$, where

$$
\left[\left[J_{\alpha}, J_{\alpha}\right]\right](X, Y)=2\left\{\left[J_{\alpha} X, J_{\alpha} Y\right]-J_{\alpha}\left[J_{\alpha} X, Y\right]-J_{\alpha}\left[X, J_{\alpha} Y\right]-[X, Y]\right\} .
$$

It is well known that the almost hypercomplex structure $H=\left(J_{\alpha}\right)$ is a hypercomplex structure if $\left[\left[J_{\alpha}, J_{\alpha}\right]\right]$ vanishes for each $\alpha=1,2,3$. Moreover it is known that one almost hypercomplex structure $H$ is hypercomplex if and only if two of the structures $J_{\alpha}(\alpha=1,2,3)$ are integrable. This means that two of the tensors $N_{\alpha}$ vanish. 1

We recall also the following definitions. Since $g$ is Hermitian metric with respect to $J_{1}$, according to 3 the class $\mathcal{W}_{4}$ is a subclass of the class of Hermitian manifolds. If $(H, G)$-manifold belongs to $\mathcal{W}_{4}$, with respect to $J_{1}$, then the almost complex structure $J_{1}$ is integrable and

$$
\begin{aligned}
F_{1}(x, y, z)=\frac{1}{2(2 n-1)}[ & g(x, y) \theta_{1}(z)-g(x, z) \theta_{1}(y) \\
& \left.-g\left(x, J_{1} y\right) \theta_{1}\left(J_{1} z\right)+g\left(x, J_{1} z\right) \theta_{1}\left(J_{1} y\right)\right],
\end{aligned}
$$

where $\theta_{1}(\cdot)=g^{i j} F_{1}\left(e_{i}, e_{j}, \cdot\right)=\delta \Phi(\cdot)$ for the basis $\left\{e_{i}\right\}_{i=1}^{4 n}$, and $\delta-$ the coderivative.

On other side the metric $g$ is a skew-Hermitian with respect to $J_{2}$ and $J_{3}$, i.e. $g\left(J_{2} x, J_{2} y\right)=g\left(J_{3} x, J_{3} y\right)=-g(x, y)$. A classification of all almost complex manifolds with skew-Hermitian metric (Norden metric or B-metric) is given in 2 . One of the basic classes of integrable almost complex manifolds 
with skew-Hermitian metric is $\mathcal{W}_{1}$. It is known that if an almost $(H, G)$ manifold belongs to $\mathcal{W}_{1}\left(J_{\alpha}\right), \alpha=2,3$, then $J_{\alpha}$ is integrable and the following equality holds

$$
\begin{aligned}
F_{\alpha}(x, y, z)=\frac{1}{4 n}[ & g(x, y) \theta_{\alpha}(z)+g(x, z) \theta_{\alpha}(y) \\
& \left.+g\left(x, J_{\alpha} y\right) \theta_{\alpha}\left(J_{\alpha} z\right)+g\left(x, J_{\alpha} z\right) \theta_{\alpha}\left(J_{\alpha} y\right)\right],
\end{aligned}
$$

where $\theta_{\alpha}(z)=g^{i j} F_{\alpha}\left(e_{i}, e_{j}, z\right), \alpha=2,3$, for an arbitrary basis $\left\{e_{i}\right\}_{i=1}^{4 n}$.

When (12) is satisfied for $(M, H, G)$, we say that $(M, H, G) \in \mathcal{W}\left(J_{1}\right)$. In the case, $(M, H, G)$ satisfies (13) for $\alpha=2$ or $\alpha=3$, we say $(M, H, G) \in$ $\mathcal{W}\left(J_{2}\right)$ or $(M, H, G) \in \mathcal{W}\left(J_{3}\right)$. Let us denote the class $\mathcal{W}:=\bigcap_{\alpha=1}^{3} \mathcal{W}\left(J_{\alpha}\right)$.

The next theorem gives a sufficient condition an almost $(H, G)$-manifold to be integrable.

Theorem $1.1\left({ }^{4}\right)$ Let $(M, H, G)$ belongs to the class $\mathcal{W}\left(J_{\alpha}\right) \cap \mathcal{W}\left(J_{\beta}\right)$. Then $(M, H, G)$ is of class $\mathcal{W}\left(J_{\gamma}\right)$ for all cyclic permutations $(\alpha, \beta, \gamma)$ of $(1,2,3)$.

Let us remark that necessary and sufficient conditions $(M, H, G)$ to be in $\mathcal{W}$ are

$$
\theta_{\alpha} \circ J_{\alpha}=-\frac{2 n}{2 n-1} \theta_{1} \circ J_{1}, \quad \alpha=2,3 .
$$

\section{Pseudo-hyper-Kähler manifolds}

Definition 2.1 (4) A pseudo-Hermitian manifold is called a pseudo-hyperKähler manifold, if $\nabla J_{\alpha}=0(\alpha=1,2,3)$ with respect to the Levi-Civita connection generated by $g$.

It is clear, then $F_{\alpha}=0(\alpha=1,2,3)$ holds or the manifold is Kählerian with respect to $J_{\alpha}$, i.e. $(M, H, G) \in \mathcal{K}\left(J_{\alpha}\right)$.

Immediately we obtain that if $(M, H, G)$ belongs to $\mathcal{K}\left(J_{\alpha}\right) \cap \mathcal{W}\left(J_{\beta}\right)$ then $(M, H, G) \in \mathcal{K}\left(J_{\gamma}\right)$ for all cyclic permutations $(\alpha, \beta, \gamma)$ of $(1,2,3)$.

Then the following sufficient condition for a $\mathcal{K}$-manifold is valid.

Theorem 2.1 (4) If $(M, H, G) \in \mathcal{K}\left(J_{\alpha}\right) \cap \mathcal{W}\left(J_{\beta}\right)$ then $M$ is a pseudo-hyperKähler manifold $(\alpha \neq \beta \in\{1,2,3\})$.

Let $\left(M^{4 n}, H, G\right)$ be a pseudo-hyper-Kähler manifold and $\nabla$ be the LeviCivita connection generated by $g$. The curvature tensor seems as follows

$$
R(X, Y) Z=\nabla_{X} \nabla_{Y} Z-\nabla_{Y} \nabla_{X} Z-\nabla_{[X, Y]} Z
$$

and the corresponding tensor of type $(0,4)$ is

$$
R(X, Y, Z, W)=g(R(X, Y) Z, W), \quad \forall X, Y, Z, W \in \mathfrak{X}(M) .
$$


Lemma 2.2 The curvature tensor of a pseudo-hyper-Kähler manifold has the following properties:

$$
\begin{aligned}
R(X, Y, Z, W) & =R\left(X, Y, J_{1} Z, J_{1} W\right)=R\left(J_{1} X, J_{1} Y, Z, W\right) \\
& =-R\left(X, Y, J_{2} Z, J_{2} W\right)=-R\left(J_{2} X, J_{2} Y, Z, W\right) \\
& =-R\left(X, Y, J_{3} Z, J_{3} W\right)=-R\left(J_{3} X, J_{3} Y, Z, W\right), \\
R(X, Y, Z, W) & =R\left(X, J_{1} Y, J_{1} Z, W\right) \\
& =-R\left(X, J_{2} Y, J_{2} Z, W\right)=-R\left(X, J_{3} Y, J_{3} Z, W\right) .
\end{aligned}
$$

Proof. The equality (17) is valid, because of (15), (16), the condition $\nabla J_{\alpha}=0(\alpha=1,2,3)$, the equality (8) and the properties of the curvature $(0,4)$-tensor.

To prove (18), we will show at first that the property $R\left(X, J_{2} Y, J_{2} Z, W\right)=$ $-R(X, Y, Z, W)$ holds. Indeed, from (17) we get

$$
R\left(J_{2} X, Y, Z, W\right)=R\left(X, J_{2} Y, Z, W\right), \quad R\left(X, Y, J_{2} Z, W\right)=R\left(X, Y, Z, J_{2} W\right)
$$

and $\mathfrak{S}_{X, Y, Z} R\left(X, Y, J_{2} Z, J_{2} W\right)=0$, where $\mathfrak{S}_{X, Y, Z}$ denotes the cyclic sum regarding $X, Y, Z$. In the last equality we replace $Y$ by $J_{2} Y$ and $W$ by $J_{2} W$. We get

$$
-R\left(X, J_{2} Y, J_{2} Z, W\right)-R\left(J_{2} Y, Z, J_{2} X, W\right)+R(Z, X, Y, W)=0 .
$$

Replacing $Y$ by $Z$, and inversely, we get

$$
-R\left(X, J_{2} Z, J_{2} Y, W\right)-R\left(J_{2} Z, Y, J_{2} X, W\right)+R(Y, X, Z, W)=0 .
$$

As we have

$$
-R\left(J_{2} Z, Y, J_{2} X, W\right)=-R\left(Z, J_{2} Y, J_{2} X, W\right)=R\left(J_{2} Y, Z, X, W\right),
$$

with the help of (19) and (20) we obtain

$$
\begin{aligned}
& -R\left(X, J_{2} Y, J_{2} Z, W\right)-R\left(X, J_{2} Z, J_{2} Y, W\right) \\
& +R(Z, X, Y, W)+R(Y, X, Z, W)=0 .
\end{aligned}
$$

According to the first Bianchi identity and (17), we obtain

$$
\begin{aligned}
-R\left(X, J_{2} Z, J_{2} Y, W\right) & =R\left(J_{2} Z, J_{2} Y, X, W\right)+R\left(J_{2} Y, X, J_{2} Z, W\right) \\
& =-R(Z, Y, X, W)-R\left(X, J_{2} Y, J_{2} Z, W\right) .
\end{aligned}
$$

Then the equality (21) seem as follows

$-2 R\left(X, J_{2} Y, J_{2} Z, W\right)+R(Z, X, Y, W)-R(X, Y, Z, W)+R(Y, Z, X, W)=0$

By the first Bianchi identity the equality is transformed in the following

$$
-2 R\left(X, J_{2} Y, J_{2} Z, W\right)-2 R(X, Y, Z, W)=0,
$$

manev12: submitted to World Scientific on August 17, 2021 
which is equivalent to

$$
R\left(X, J_{2} Y, J_{2} Z, W\right)=-R(X, Y, Z, W) .
$$

As the tensor $R$ has the same properties with respect to $J_{3}$, and to $J_{2}$, it follows that the next equality holds, too.

$$
R\left(X, J_{3} Y, J_{3} Z, W\right)=-R(X, Y, Z, W) .
$$

Using (22) and (23) for $J_{1}=J_{2} J_{3}$ we get successively that

$$
\begin{aligned}
R(X, Y, Z, W) & =R\left(X, J_{1} Y, J_{1} Z, W\right) \\
& =R\left(X, J_{2}\left(J_{3} Y\right), J_{2}\left(J_{3} Z\right), W\right)=-R\left(X, J_{3} Y, J_{3} Z, W\right),
\end{aligned}
$$

which completes the proof of (18).

Now we will prove a theorem which gives us a geometric characteristic of the pseudo-hyper-Kähler manifolds.

Theorem 2.3 Each pseudo-hyper-Kähler manifold is a flat pseudo-Riemannian manifold with signature $(2 n, 2 n)$.

Proof. Lemma 2.2 implies the properties

$$
\begin{aligned}
-R(X, Y, Z, W) & =R\left(X, J_{1} Y, Z, J_{1} W\right) \\
& =R\left(X, J_{2} Y, Z, J_{2} W\right)=R\left(X, J_{3} Y, Z, J_{3} W\right) .
\end{aligned}
$$

As $J_{1}=J_{2} J_{3}$, we also have the following

$$
\begin{aligned}
R\left(X, J_{1} Y, Z, J_{1} W\right) & =R\left(X, J_{2}\left(J_{3} Y\right), Z, J_{2}\left(J_{3} W\right)\right) \\
& =-R\left(X, J_{3} Y, Z, J_{3} W\right)=R(X, Y, Z, W) .
\end{aligned}
$$

Comparing (24) with the last equality we receive

$$
-R(X, Y, Z, W)=R\left(X, J_{1} Y, Z, J_{1} W\right)=R(X, Y, Z, W),
$$

or $R \equiv 0$.

\section{Conformal transformations of the pseudo-Hermitian metric}

The usual conformal transformation $c: \bar{g}=e^{2 u} g$, where $u$ is a differential function on $M^{4 n}$, is known. Since $g_{\alpha}(\cdot, \cdot)=g\left(J_{\alpha} \cdot, \cdot\right)$, the conformal transformation of $g$ causes the same changes of the pseudo-Hermitian metrics $g_{2}, g_{3}$ and the Kähler form $\Phi \equiv g_{1}$. Then we say that it is given a conformal transformation $c$ of $G$ to $\bar{G}$ determined by $u \in \mathcal{F}(M)$. These conformal transformations form a group denoted by $C$. The hypercomplex pseudo-Hermitian manifolds $(M, H, G)$ and $(M, H, \bar{G})$ we call $C$-equivalent manifolds or conformalequivalent manifolds. 
Let $\nabla$ and $\bar{\nabla}$ be the Levi-Civita connections determined by the metrics $g$ and $\bar{g}$, respectively. The known condition for a Levi-Civita connection implies the following relation

$$
\bar{\nabla}_{X} Y=\nabla_{X} Y+\mathrm{d} u(X) Y+\mathrm{d} u(Y) X-g(X, Y) \operatorname{grad}(u) .
$$

Using (25) and the definitions of structural tensors for $\nabla$ and $\bar{\nabla}$ we obtain

$$
\begin{array}{r}
\bar{F}_{1}(X, Y, Z)=e^{2 u}\left[F_{1}(X, Y, Z)-g(X, Y) \mathrm{d} u\left(J_{1} Z\right)+g(X, Z) \mathrm{d} u\left(J_{1} Y\right)\right. \\
\left.+g\left(J_{1} X, Y\right) \mathrm{d} u(Z)-g\left(J_{1} X, Z\right) \mathrm{d} u(Y)\right], \\
\bar{F}_{\alpha}(X, Y, Z)=e^{2 u}\left[F_{\alpha}(X, Y, Z)+g(X, Y) \mathrm{d} u\left(J_{\alpha} Z\right)+g(X, Z) \mathrm{d} u\left(J_{\alpha} Y\right)\right. \\
\left.-g\left(J_{\alpha} X, Y\right) \mathrm{d} u(Z)-g\left(J_{\alpha} X, Z\right) \mathrm{d} u(Y)\right]
\end{array}
$$

for $\alpha=2,3$. The last two equalities imply the following relations for the corresponding structural 1-forms

$$
\bar{\theta}_{1}=\theta_{1}-2(2 n-1) \mathrm{d} u \circ J_{1}, \quad \bar{\theta}_{\alpha}=\theta_{\alpha}+4 n \mathrm{~d} u \circ J_{\alpha}, \quad \alpha=2,3 .
$$

Let us denote the following $(0,3)$-tensors.

$$
\begin{gathered}
P_{1}(x, y, z)=F_{1}(x, y, z) \\
-\frac{1}{2(2 n-1)}\left[g(x, y) \theta_{1}(z)-g(x, z) \theta_{1}(y)\right. \\
\left.-g\left(x, J_{1} y\right) \theta_{1}\left(J_{1} z\right)+g\left(x, J_{1} z\right) \theta_{1}\left(J_{1} y\right)\right], \\
P_{\alpha}(x, y, z)=F_{\alpha}(x, y, z) \quad \\
-\frac{1}{4 n}\left[g(x, y) \theta_{\alpha}(z)+g(x, z) \theta_{\alpha}(y)\right. \\
\left.\quad+g\left(x, J_{\alpha} y\right) \theta_{\alpha}\left(J_{\alpha} z\right)+g\left(x, J_{\alpha} z\right) \theta_{\alpha}\left(J_{\alpha} y\right)\right], \quad \alpha=2,3 .
\end{gathered}
$$

According to (12) and (13) it is clear that

$$
(M, H, G) \in \mathcal{W}\left(J_{\alpha}\right) \Longleftrightarrow P_{\alpha}=0 \quad(\alpha=1,2,3) .
$$

The equalities (26)-(28) imply the following two interconnections

$$
\begin{gathered}
\bar{P}_{\alpha}=e^{2 u} P_{\alpha}, \quad \alpha=1,2,3 ; \\
\bar{\theta}_{\alpha} \circ J_{\alpha}+\frac{2 n}{2 n-1} \bar{\theta}_{1} \circ J_{1}=\theta_{\alpha} \circ J_{\alpha}+\frac{2 n}{2 n-1} \theta_{1} \circ J_{1}, \quad \alpha=2,3 .
\end{gathered}
$$

From (31) we receive that each of $\mathcal{W}\left(J_{\alpha}\right)(\alpha=1,2,3)$ is invariant with respect to the conformal transformations of $C$, i.e. they are $C$-invariant classes. Having in mind also (32), we state the validity of the following Theorem 3.1 The class $\mathcal{W}$ of hypercomplex pseudo-Hermitian manifolds is C-invariant.

Now we will determine the class of the (locally) $C$-equivalent $\mathcal{K}$-manifolds. Let us denote the following subclass $\mathcal{W}^{0}:=\left\{\mathcal{W} \mid d\left(\theta_{1} \circ J_{1}\right)=0\right\}$. 
Theorem 3.2 A hypercomplex pseudo-Hermitian manifold belongs to $\mathcal{W}^{0}$ if and only if it is C-equivalent to a pseudo-hyper-Kähler manifold.

Proof. Let $(M, H, G)$ be a pseudo-hyper-Kähler manifold, i.e. $(M, H, G) \in \mathcal{K}$. Then $F_{\alpha}=\theta_{\alpha}=0(\alpha=1,2,3)$. Hence (28) has the form

$$
\bar{\theta}_{1}=-2(2 n-1) \mathrm{d} u \circ J_{1}, \quad \bar{\theta}_{\alpha}=4 n \mathrm{~d} u \circ J_{\alpha}, \quad \alpha=2,3 .
$$

From (26), (27) and (33) and having in mind (12) and (13) we obtain that $(M, H, \bar{G})$ is a $\mathcal{W}$-manifold. According to (33) the 1 -forms $\bar{\theta}_{\alpha} \circ J_{\alpha}(\alpha=1,2,3)$ are closed. Because of (14) the condition $\mathrm{d}\left(\bar{\theta}_{1} \circ J_{1}\right)=0$ is sufficient.

Conversely, let $(M, H, \bar{G})$ be a $\mathcal{W}$-manifold with closed $\bar{\theta}_{1} \circ J_{1}$. Because of (14) the 1-forms $\bar{\theta}_{\alpha} \circ J_{\alpha}(\alpha=2,3)$ are closed, too. We determine the function $u$ as a solution of the differential equation $\mathrm{d} u=-\frac{1}{2(2 n-1)} \bar{\theta}_{1} \circ J_{1}$. Then by an immediate verification we state that the transformation $c^{-1}: g=e^{-2 u} \bar{g}$ converts $(M, H, \bar{G})$ into $(M, H, G) \in \mathcal{K}$. This completes the proof.

Let us remark the following inclusions

$$
\mathcal{K} \subset \mathcal{W}^{0} \subset \mathcal{W} \subset \mathcal{W}\left(J_{\alpha}\right), \quad \alpha=1,2,3 .
$$

Let $R, \rho, \tau$ and $\bar{R}, \bar{\rho}, \bar{\tau}$ be the curvature tensors, the Ricci tensors, the scalar curvatures corresponding to $\nabla$ and $\bar{\nabla}$, respectively. The following tensor is curvature-like, i.e. it has the same properties as $R$.

$$
\begin{aligned}
\psi_{1}(S)(X, Y, Z, U)= & g(Y, Z) S(X, U)-g(X, Z) S(Y, U) \\
& +g(X, U) S(Y, Z)-g(Y, U) S(X, Z),
\end{aligned}
$$

where $S$ is a symmetric tensor.

Having in mind (25) and (15), we obtain

Proposition 3.3 The following relations hold for the $C$-equivalent $(H, G)$ manifolds

$$
\begin{gathered}
\bar{R}=e^{2 u}\left\{R-\psi_{1}(S)\right\}, \\
\bar{\rho}=\rho-\operatorname{tr} S g-2(2 n-1) S, \quad \bar{\tau}=e^{-2 u}\{\tau-2(4 n-1) \operatorname{tr} S\},
\end{gathered}
$$

where

$$
S(Y, Z)=S(Z, Y)=\left(\nabla_{Y} \mathrm{~d} u\right) Z+\mathrm{d} u(Y) \mathrm{d} u(Z)-\frac{1}{2} \mathrm{~d} u(\operatorname{grad}(\mathrm{d} u)) g(Y, Z) .
$$

If $(M, H, G)$ is a $C$-equivalent $\mathcal{W}$-manifold to a $\mathcal{K}$-manifold, i.e. $(M, H, G) \in \mathcal{W}^{0}$, then Proposition 3.3 implies

Corollary 3.4 $A \mathcal{W}^{0}$-manifold has the following curvature characteristic

$$
\begin{gathered}
R=\frac{1}{2(2 n-1)}\left\{\psi_{1}(\rho)-\frac{\tau}{4 n-1} \pi_{1}\right\}, \\
\text { where } \pi_{1}(X, Y, Z, U)=\frac{1}{2} \psi_{1}(g)=g(Y, Z) g(X, U)-g(X, Z) g(Y, U) .
\end{gathered}
$$


It is well known that the $C$-invariant tensor of each pseudo-Riemannian manifold is the so-called Weil tensor $W$. From (34) we receive immediately

$$
\bar{W}=e^{2 u} W, \quad W=R-\frac{1}{2(2 n-1)}\left\{\psi_{1}(\rho)-\frac{\tau}{4 n-1} \pi_{1}\right\} .
$$

Let us remark that the vanishing of the Weil tensor is a necessary and sufficient condition a pseudo-Riemannian manifold to be conformal equivalent to a flat manifold with dimension greater than 3 .

This is confirmed by the combining of Theorem 2.3 . Theorem 3.2 and Corollary 3.4. i.e. $(M, H, G) \in \mathcal{W}^{0}$ iff $W=0$ on $(M, H, G)$.

Since each conformal transformation determines uniquely a symmetric tensor $S$ by (35) then it takes an interest in the consideration $S$ as a bilinear form on $T_{p} M$ belonging to each of the components $L_{\alpha},(\alpha=0,1,2,3)$.

Let $S \in L_{0}$. In view of (5) $\operatorname{tr} S=0$ holds and according to (34) we receive $\bar{\tau}=e^{-2 u} \tau$ and an invariant tensor $W_{0}=R-\frac{1}{2(2 n-1)} \psi_{1}(\rho)$. When $W_{0}$ vanishes on $(M, H, G)$ then the curvature tensor has the form $R=\frac{1}{2(2 n-1)} \psi_{1}(\rho)$.

In the cases when $S \in L_{\alpha}(\alpha=1,2,3)$ we consider $(M, H, G)$ as an $\mathcal{W}^{0}$ manifold. Then according to Theorem 2.3 and Theorem 3.2 we have $\bar{R}=0$ on the $C$-equivalent $\mathcal{K}$-manifold of $(M, H, G)$.

Now let $S \in L_{1}$. By reason of $g \in L_{1}$ we have a cause for the consideration of the possibility $S=\lambda g$. Hence $\lambda=\frac{\operatorname{tr} S}{4 n}=\frac{\tau}{8 n(4 n-1)}$. Then having in mind (34) $R=\frac{\tau}{4 n(4 n-1)} \pi_{1}$ holds true. From here it is clear that if $S \in L_{1}$ then $(M, H, G)$ is an Einstein manifold.

Let us consider the case when $S \in L_{2}$. Then according to (6) $\operatorname{tr} S$ vanishes, and from (34) $\tau$ vanishes, too. Because of $g_{3} \in L_{2}$ we consider $S=\lambda g_{3}$, whence $\lambda=-\frac{\operatorname{tr}\left(S \circ J_{3}\right)}{4 n}$. Then (34) implies $R=\frac{\operatorname{tr}\left(S \circ J_{3}\right)}{4 n} \pi_{3}^{J_{3}}$, where $\pi_{3}^{J_{3}}$ is the following tensor $\pi_{3}$ with respect to the complex structure $J=J_{3}$

$$
\pi_{3}(X, Y, Z, U)=-\pi_{1}(X, Y, J Z, U)-\pi_{1}(X, Y, Z, J U) .
$$

It is known ${ }^{2}$ that $\pi_{3}$ is a Kähler curvature-like tensor, i.e. it satisfies the property $\pi_{3}(X, Y, J Z, J U)=-\pi_{3}(X, Y, Z, U)$. Therefore in this case $R$ is Kählerian with respect to $J_{3}$ and the tensor $R^{* J_{3}}: R^{* J_{3}}(X, Y, Z, U)=R\left(X, Y, Z, J_{3} U\right)$ is curvature-like. Then we obtain immediately

$$
R=\frac{\tau\left(R^{* J_{3}}\right)}{8 n(2 n-1)} \pi_{3}^{J_{3}}, \quad \rho=-\frac{\tau\left(R^{* J_{3}}\right)}{4 n} g_{3} .
$$

Hence if $S \in L_{2}$ then $(M, H, G)$ is a ${ }^{*}$-Einstein manifold with respect to $J_{3}$.

By an analogous way, in the case when $S \in L_{3}$ we receive that $(M, H, G)$ is a *-Einstein manifold with respect to $J_{2}$.

manev12: submitted to World Scientific on August 17, 2021 


\section{A 4-dimensional pseudo-Riemannian spherical manifold with $(H, G)$-structure}

In 4 is considered a hypersurface $S_{2}^{4}$ in $\mathbb{R}_{2}^{5}$ by the equation

$$
-\left(z^{1}\right)^{2}-\left(z^{2}\right)^{2}+\left(z^{3}\right)^{2}+\left(z^{4}\right)^{2}+\left(z^{5}\right)^{2}=1,
$$

where $Z\left(z^{1}, z^{2}, z^{3}, z^{4}, z^{5}\right)$ is the positional vector of $p \in S_{2}^{4}$.

Let $\left(u^{1}, u^{2}, u^{3}, u^{4}\right)$ be local coordinates of $p$ on $S_{2}^{4}$. The hypersurface $S_{2}^{4}$ is defined by the scalar parametric equations:

$$
\begin{gathered}
z^{1}=\sinh u^{1} \cos u^{2}, \quad z^{2}=\sinh u^{1} \sin u^{2}, \quad z^{3}=\cosh u^{1} \cos u^{3} \cos u^{4}, \\
z^{4}=\cosh u^{1} \cos u^{3} \sin u^{4}, \quad z^{5}=\cosh u^{1} \sin u^{3} .
\end{gathered}
$$

Further we consider the manifold on $\tilde{S}_{2}^{4}=S_{2}^{4} \backslash\{(0,0,0,0, \pm 1)\}$, i.e. we omit two points for which $\left\{u^{1} \neq 0\right\} \cap\left\{u^{3} \neq(2 k+1) \pi / 2, k \in \mathbb{Z}\right\}$. The tangent space $T_{p} \tilde{S}_{2}^{4}$ of $\tilde{S}_{2}^{4}$ in the point $p \in \tilde{S}_{2}^{4}$ is determined by the vectors $z_{i}=\frac{\partial Z}{\partial u^{i}}(i=$ $1,2,3,4)$. The vectors $z_{i}$ are linearly independent on $\tilde{S}_{2}^{4}$, defined by (38), and $T_{p} \tilde{S}_{2}^{4}$ has a basis $\left(z_{1}, z_{2}, z_{3}, z_{4}\right)$ in every point $p \in \tilde{S}_{2}^{4}$.

The restriction of $\langle\cdot, \cdot\rangle$ from $\mathbb{R}_{2}^{5}$ to $S_{2}^{4}$ is a pseudo-Riemannian metric $g$ on $S_{2}^{4}$ with signature $(2,2)$. The non-zero components $g_{i j}=\left\langle z_{i}, z_{j}\right\rangle$ are

$$
g_{11}=-1, g_{22}=-\sinh ^{2} u^{1}, g_{33}=\cosh ^{2} u^{1}, g_{44}=\cosh ^{2} u^{1} \cos ^{2} u^{3} .
$$

The hypersurface $S_{2}^{4}$ is equipped with an almost hypercomplex structure $H=\left(J_{\alpha}\right),(\alpha=1,2,3)$, where the non-zero components of the matrix of $J_{\alpha}$ with respect to the local basis $\left\{\frac{\partial}{\partial u^{i}}\right\}_{i=1}^{4}$ are

$$
\begin{array}{ll}
\left(J_{1}\right)_{2}^{1}=-\frac{1}{\left(J_{1}\right)_{1}^{2}}=-\sinh u^{1}, & \left(J_{1}\right)_{4}^{3}=-\frac{1}{\left(J_{1}\right)_{3}^{4}}=\cos u^{3}, \\
\left(J_{2}\right)_{3}^{1}=-\frac{1}{\left(J_{2}\right)_{1}^{3}}=-\cosh u^{1}, & \left(J_{2}\right)_{4}^{2}=-\frac{1}{\left(J_{2}\right)_{2}^{4}}=-\operatorname{coth} u^{1} \cos u^{3}, \\
\left(J_{3}\right)_{4}^{1}=-\frac{1}{\left(J_{3}\right)_{1}^{4}}=\cosh u^{1} \cos u^{3}, & \left(J_{3}\right)_{2}^{3}=-\frac{1}{\left(J_{3}\right)_{3}^{2}}=\tanh u^{1} .
\end{array}
$$

Theorem $4.1\left({ }^{4}\right)$ The spherical pseudo-Riemannian 4-dimensional manifold, defined by (38), admits a hypercomplex pseudo-Hermitian structure on $\tilde{S}_{2}^{4}$, determined by (40) and (39), with respect to which it is of the class $\mathcal{W}\left(J_{1}\right)$ but it does not belong to $\mathcal{W}$ and it has a constant sectional curvature $k=1$.

Let us consider a conformal transformation determined by the function $u$ which is a solution of the equation $\mathrm{d} u=-\frac{1}{2(2 n-1)}\left(\theta_{1} \circ J_{1}\right)$, where the nonzero component of $\theta_{1}$ with respect to the local basis $\left\{\frac{\partial}{\partial u^{i}}\right\}(i=1,2,3,4)$ is $\theta_{1}\left(\frac{\partial}{\partial u^{2}}\right)=\frac{2 \sinh ^{2} u^{1}}{\cosh u^{1}}$.

Since $\tilde{S}_{2}^{4}$ has a constant sectional curvature then the Weil tensor is vanishes, i.e. $\tilde{S}_{2}^{4}$ is $C$-equivalent to a flat $\mathcal{K}\left(J_{1}\right)$-manifold. If we admit that it is in 
$\mathcal{K}$, then according to Theorem 3.2 we obtain that the manifold $\left(\tilde{S}_{2}^{4}, H, G\right) \in \mathcal{W}$ which is a contradiction. Therefore the considered manifold is $C$-equivalent to a flat $\mathcal{K}\left(J_{1}\right)$-manifold, but it is not a pseudo-hyper-Kähler manifold. By direct verification we state that the tensor $S$ of this conformal transformation belongs to $L_{1}$. Therefore $\left(\tilde{S}_{2}^{4}, H, G\right)$ is an Einstein manifold.

\section{References}

1. D. V. Alekseevsky and S. Marchiafava, Quaternionic structures on a manifold and subordinated structures, Ann. Mat. Pura Appl. (IV), CLXXI (1996), 205-273.

2. G. Ganchev and A. Borisov, Note on the almost complex manifolds with a Norden metric, Compt. rend. Acad. bulg. Sci., 39 (1986), no. 5, 31-34.

3. A. Gray and L. M. Hervella, The sixteen classes of almost Hermitian manifolds and their linear invariants, Ann. Mat. Pura Appl. (IV), CXXIII (1980), 35-58.

4. K. Gribachev, M. Manev and S. Dimiev, Almost hypercomplex pseudoHermitian manifolds, Boll. Unione Mat. Ital. Sez. A, (to appear)

5. A. Sommese, Quaternionic manifolds, Math. Ann. 212 (1975), 191-214.

6. J. Wolf, Spaces of constant curvature, University of California, Berkley, California, 1972. 